\title{
MGRF Controlled Stochastic Deformable Model
}

\author{
Ayman El-Baz ${ }^{1}$, Aly Farag ${ }^{1}$, and Georgy Gimelfarb ${ }^{2}$ \\ 1 Computer Vision and Image Processing Laboratory, \\ University of Louisville, Louisville, KY 40292, USA \\ ffarag, elbaz\}@cvip.Louisville.edu \\ http://www.cvip.louisville.edu \\ 2 Department of Computer Science, Tamaki Campus, \\ University of Auckland, Auckland, New Zealand
}

\begin{abstract}
Deformable or active contour, and surface models are powerful image segmentation techniques. We introduce a novel fast and robust bi-directional parametric deformable model which is able to segment regions of intricate shape in multi-modal greyscale images. The power of the algorithm in terms of computation time and robustness is owing to the use of joint probabilities of the signals and region labels in individual points as external forces guiding the model evolution. These joint probabilities are derived from a Markov-Gibbs random field (MGRF) image model considering an image as a sample of two interrelated spatial stochastic processes. The low level process with conditionally independent and arbitrarily distributed signals relates to the observed image whereas its hidden map of regions is represented with the high level MGRF of interdependent region labels. Marginal probability distributions of signals in each region are recovered from a mixed empirical signal distribution over the whole image. In so doing, each marginal is approximated with a linear combination of Gaussians (LCG) having both positive and negative components. The LCG parameters are estimated using our previously proposed modification of the EM algorithm, and the high-level Gibbs potentials are computed analytically. Comparative experiments show that the proposed model outlines complicated boundaries of different modal objects much more accurately than other known counterparts.
\end{abstract}

\section{Introduction}

Deformable or active model, is a curve in a $2 \mathrm{D}$ digital image or a surface in a $3 \mathrm{D}$ image that evolves to outline a desired object. The evolution is controlled by internal and external forces combined, together with user defined constraints, into internal and external energy terms, respectively. Introduced first by Kass et al. 1, the models gave rise to one of the most dynamic and successful research areas in edge detection, image segmentation, shape modeling, and visual tracking. By representation and implementation, deformable models are broadly categorized into parametric (e.g. [1, 2, ) and geometric (e.g. [3, 4]) classes. In this 
paper, we focus on parametric deformable models that form a parametric curve and move it toward an object boundary.

Performance of the deformable model depends on proper initialization, efficiency of the energy minimization process, and adequate selection of the force functions and energy functions. The original iterative minimization in 1 is based on a closed-form solution of Eulerian differential equations specifying the desired minimum. But it turns out to be unstable and usually gets trapped into local minima. Amini et al. [5] point to shortcomings of this minimization process in [1] and improve it by representing a contour as a linked chain of control points and minimizing its total energy by discrete dynamic programming. This approach allows for rigid constraints on the energy function that make the minimization more stable. But still its control parameters must be adjusted very carefully, and the process remains too time consuming. Time complexity of a more advanced greedy algorithm proposed by Williams and Shah 6] is linear with respect to both the number of control points and the neighbors of each point which are taken into account for energy minimization. It is much more stable and is simultaneously more than an order of magnitude faster than the previous techniques.

To more closely approach a complicated boundary with concavities, Grzeszczuk and Levin [7] control snake evolution by simulated annealing. In principle the latter eventually reaches the global minimum of energy and can escape local traps. But in practice, as emphasized in [8], simulated annealing typically stops very far from the global minimum. Alternative minimum-cut graph algorithms in 9] guarantee a close approximation of geodesic contours in 2D (or minimal surface in 3D) images having the minimum global energy under an arbitrary Riemannian metric of a set of boundary conditions. But both these minimization processes are extremely slow. A faster precise boundary approximation proposed by $\mathrm{Xu}$ and Prince [2] introduces a gradient vector flow (GVF) as a new external force. Due to its larger capture range, the GVF allows a contour to move into the boundary concavities. A bi-directional deformable model in 4] combines the geodesic contour and the GVF.

In spite of good segmentation results for objects of relatively simple shapes, the above conventional deformable models have serious drawbacks. Most of them are slow compared to other segmentation techniques, and the model evolution frequently stops well before approaching a complicated object boundary with concavities. Also, to initialize the model, typically a closed curve has to be interactively drawn near the desired boundary, and this manual step hinders their use in many applications.

In this paper we propose joint probabilities of signals and region labels in individual image points as a new class of external forces to guide the model evolution. This class overcomes the above problems in the case of multi-modal images where each object of interest relates to a separate mode of the empirical marginal signal distribution. We call a model with the probabilistic external forces a stochastic deformable model. Its advantages over more conventional models are in the automated initialization, insensitivity to the initialization, and ability to follow complex shapes with concavities. 


\section{Parametric Deformable 2D Contours}

A conventional parametric deformable 2D model, or snake, is a curve $\Phi=$ $(\phi(\tau)=(u(\tau), v(\tau)) ; \tau \in T)$ in planar Cartesian co-ordinates $(u, v)$ where $\tau$ is the continuous or discrete index of a contour point and $T$ is the index range. The deformable model moves through the spatial image domain to minimize the total energy

$$
E=E_{\mathrm{int}}+E_{\mathrm{ext}}=\int_{\tau \in T} \xi_{\text {int }}(\phi(\tau))+\xi_{\mathrm{ext}}(\phi(\tau)) d \tau
$$

where $\xi_{\text {int }}(\phi(\tau))$ and $\xi_{\text {ext }}(\phi(\tau))$ denote the internal and external forces, respectively, that control the point-wise model movements. The total energy is the sum of two terms, the internal energy keeping the deformable model as a single unit and the external one attracting the model to the region boundary. The internal force is typically defined as $\xi_{\text {int }}(\phi(\tau))=\alpha\left|\phi^{\prime}(\tau)\right|^{2}+\beta\left|\phi^{\prime \prime}(\tau)\right|^{2}$ where weights $\alpha$ and $\beta$ control the curve's tension and rigidity, respectively, and $\phi^{\prime}(\tau)$ and $\phi^{\prime \prime}(\tau)$ are the first and second derivatives of $\phi(\tau)$ with respect to $\tau$.

Typical external forces designed in [1] to lead an active contour toward step edges in a grayscale image $\mathbf{Y}$ are:

$$
\begin{aligned}
\xi_{\text {ext }}(\phi(\tau))= & -|\nabla \mathbf{Y}(\phi(\tau))|^{2} \text { or } \\
& -|\nabla[G(\phi(\tau)) * \mathbf{Y}(\phi(\tau))]|^{2}
\end{aligned}
$$

where $G(\ldots)$ is a 2D Gaussian kernel and $\nabla$ denotes the gradient operator. But both these and other traditional external forces (e.g. based on lines, edges, or the GVF) fail to make the contour to closely approach an intricate boundary with concavities. Moreover, due to high computational complexity the deformable models with most of such external energies are slow compared to the other segmentation techniques.

\section{Stochastic Deformable 2D Contour}

The above drawbacks are overcome to a large extent when joint probabilities of image signals and region labels in individual points along the deformable model are used as new external forces. The probabilities are easily derived from a simple MGRF model of multi-modal greyscale images. The model merges two interrelated spatial stochastic processes. The low level process is a conditionally independent random field of image signals (gray levels) with arbitrary probability distributions of signals. The distributions differ for different regions but are the same for each pixel in the region. By assumption, their mixed distribution for the whole image is multi-modal, each mode corresponding to one of the regions. This process relates to the observed image whereas a hidden map of regions is represented with the high level MGRF of interdependent region labels. The interdependence is restricted to only pairs of labels in the nearest 8-neighborhood of each 
pixel. By symmetry considerations, Gibbs potentials are the same for all pairs and regions, depending only on whether the labels are equal or not in the pair, and thus have only two values: $\gamma$ for the equal and $-\gamma$ for unequal pairs of labels.

To compute the forces, the low-level model is identified for a given image $\mathbf{Y}$ by the LCG-approximation of the conditional marginal signal distributions in each region with the modified EM-algorithm proposed in [10. The estimated distributions allow us to get a region map $\mathbf{X}$ for the image $\mathbf{Y}$ by classifying the individual pixels. Then the high-level model is identified for the region map $\mathbf{X}$ using the analytic potential estimate derived in accordance with [11. In our case, $\gamma=\frac{K^{2}}{(K-1)}\left(f_{\text {eq }}(\mathbf{X})-\frac{1}{K}\right)$ where $K$ is the number of modes, or regions in the image $\mathbf{Y}$ and $f_{\text {eq }}(\mathbf{X})$ denotes the empirical frequency of the equal labels in the pairs of the nearest 8-neighboring pixels in the map $\mathbf{X}$. The total energy of the active contour is minimized by exploiting the greedy strategy [6]. The detailed description of these force computations has been given in our technical report 12 .

Let $k$ and $q$ denote a region label and a gray level, respectively: $k=1, \ldots, K$. The stochastic external force for each control point $\phi(\tau)$ of a current deformable contour evolving in a region $k^{*}$ is defined as follows:

$$
\xi_{\text {ext }}(\phi(\tau))=\left\{\begin{array}{r}
-p(q \mid k) p(k) \text { if } k=k^{*} \\
p(q \mid k) p(k) \text { if } k \neq k^{*}
\end{array}\right.
$$

where $q=\mathbf{Y}(\phi(\tau))$ and $k=\mathbf{X}(\phi(\tau))$. For each iteration of the greedy algorithm, the neighborhood of each control point $\phi(\tau)$ is analyzed, and the neighboring pixel ensuring the smallest total energy becomes the new position for that control point as shown in Fig. 1. The iterations continue until the whole deformable model (that is, all its current control points) do not change anymore. The proposed algorithm of segmenting the region $k^{*}$ is as follows:

1. Collect the empirical gray level distribution for a given image $\mathbf{Y}$ and identify the low level MGRF model 10.

2. Use the Bayesian classifier to get the map $\mathbf{X}$ and identify the high level MGRF model (i.e. find $\gamma$ ).

3. Use the pixel with the maximum joint probability $p\left(q, k^{*}\right)$ as an automatic seed to initialize the deformable contour.

4. For each control point $\phi(\tau)$ on the current deformable contour, calculate sign distances indicating exterior $(+)$ or interior $(-)$ positions of each of the eight nearest neighbors w.r.t. the contour as shown in Fig. 11.

5. Check the label $k=\mathbf{X}(\phi(\tau))$ for each control point:

(a) If the point is assigned to the region $k=k^{*}$, then

i. Estimate the region labels for its neighbors such that they have the $(+)$ distance.

ii. If some of these sites are also assigned to the class $k^{*}$, then move the control point to the neighboring position ensuring the minimum total energy (i.e., expand the contour).

iii. Otherwise, do not move this point (the steady state). 
(b) If the point is assigned to the region $k \neq k^{*}$, then

i. Estimate the region labels for its neighbors such that they have the (-) distance.

ii. Move the control point to the neighboring position ensuring the minimum total energy (i.e. contract the contour)

6. If the iteration adds new control points, use the cubic spline interpolation of the whole contour and then smooth all its control points with a low pass filter.

7. Repeat steps 4, 5, and 6] until no positional changes in the control points occur.

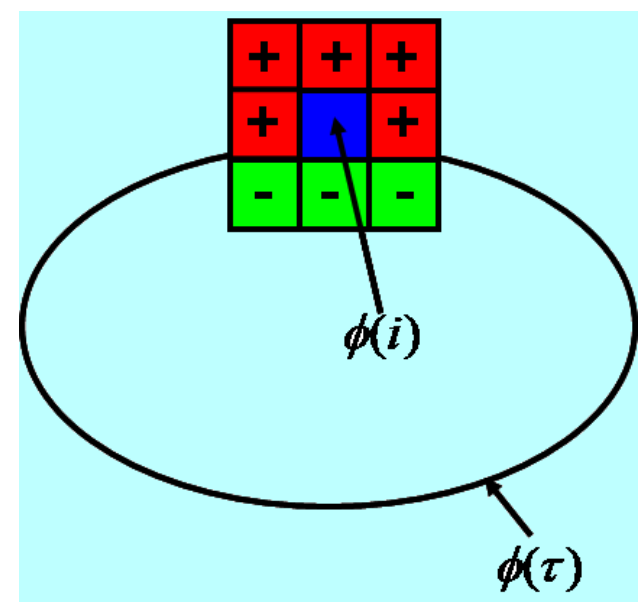

Fig. 1. Greedy propagation of the deformable model

\section{Experiments and Conclusions}

To assess robustness and computational performance, the proposed model has been tested on images of different objects with intricate shapes such as "Sea star" in Fig. 2(a). The image has only two dominant modes $(K=2)$ : the darker background and the brighter object. The low level model is identified by the modified EM algorithm [10]. Figures 2 (b)-(d) show, respectively, the LCG approximation of the mixed empirical bi-modal distribution of signals for the image, the individual components of the LCG, and the LCG-models for each region. Pixelwise Bayesian classification based on this latter model produces the initial region map for the "Sea star" image. Then the Gibbs potentials are analytically estimated [1] (in this case $\gamma=2.17$ ), and the identified MGRF model is used to select the point with maximum joint signal/label probability to initialize the deformable contour. Figure 3(a) shows the initialization with a circle having the radius of 20 pixels from the maximum probable point. Figure 3(b) shows the 


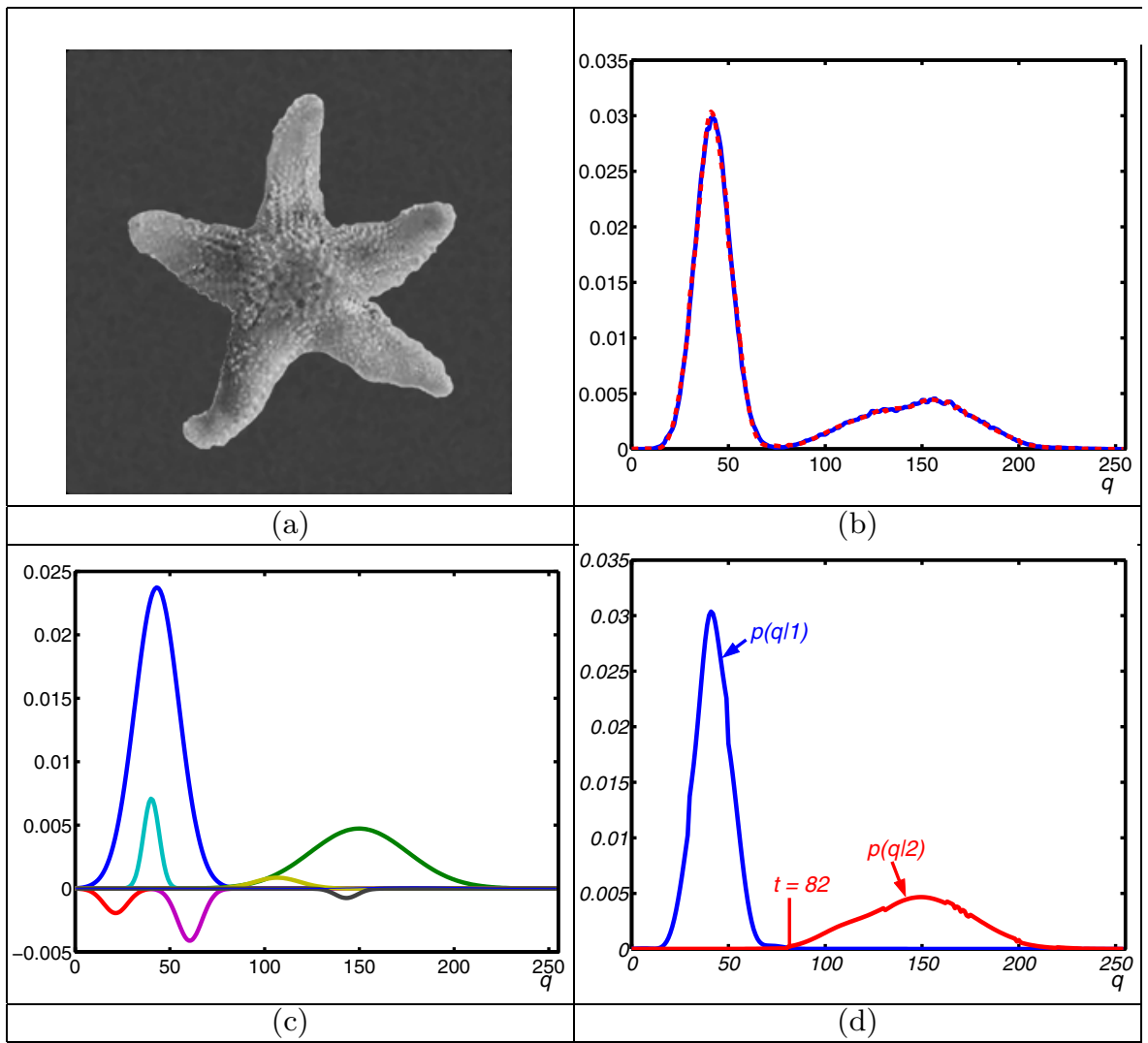

Fig. 2. "Sea star" (a), LCG approximation of the empirical distribution (b), individual Gaussians (c), and LCG region models (d)

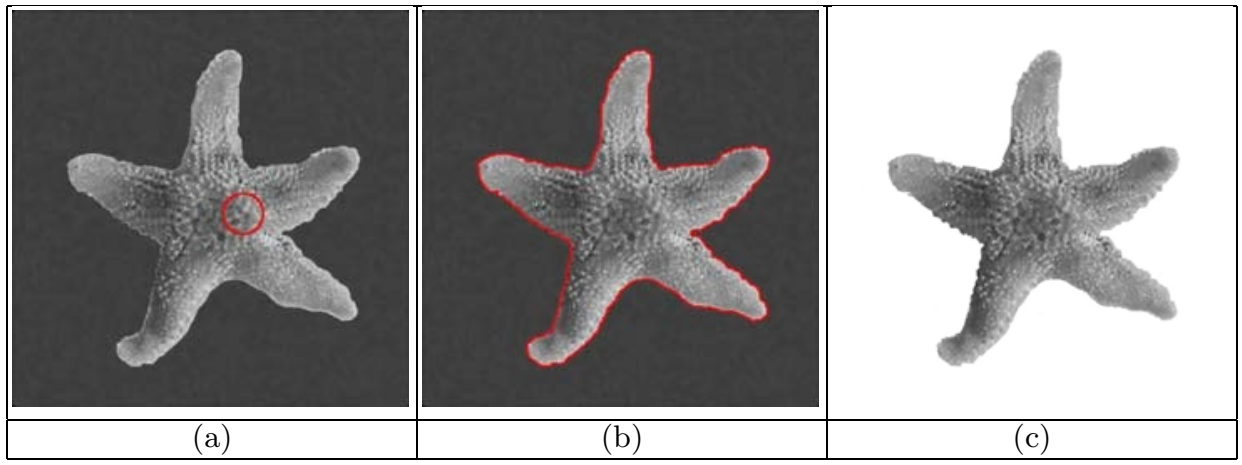

Fig. 3. Initialization of the deformable contour (a), our segmentation with the error of $0.0036 \%$ (b), and the ground truth (c) 


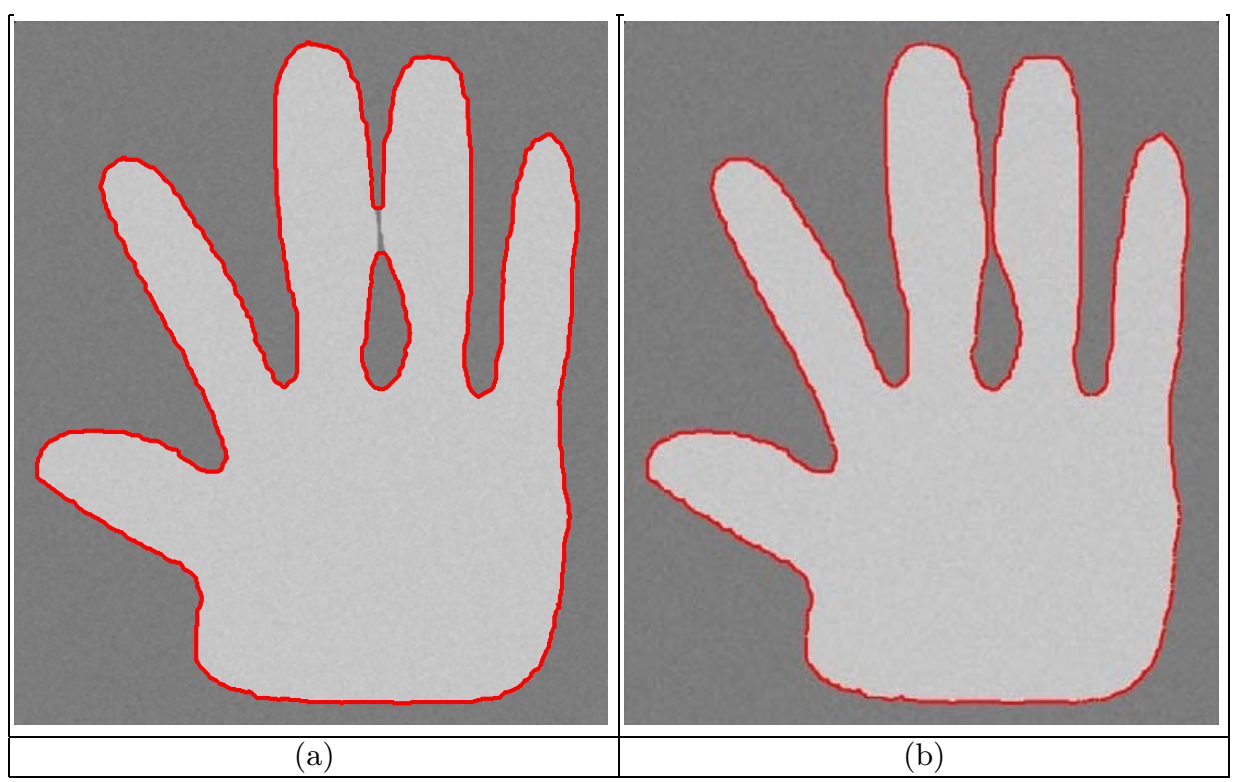

Fig. 4. GAC [13] (a) and our (b) segmentation of "Hand"

"Sea star" region segmented with the proposed deformable model. The segmentation error is $0.0036 \%$ with respect to the ground truth in Fig. 3)(c).

Figure 4 compares results of a popular geometric model, the geodesic active contour (GAC) [13, and our parametric model for a hand-shaped object. Our model preserves better the topology of the shape. Because two middle fingers are very close to each other, the initial curve splits into two separate curves so that the final GAC consists of a larger outer curve and a disjointed smaller inner curve shown in Fig. 4(a). Our segmentation in Fig. 4(b) keeps the separate boundary of each finger, and the final contour correctly reflects the shape of the hand.

Figure 5 highlights the advantages of our stochastic model over two conventional parametric deformable models by comparing segmentation results obtained by the proposed approach and with the greedy algorithm using the conventional deformable model proposed in [1] and with the like algorithm proposed in 2. The two latter deformable models involve the image gradient (DMG) and the gradient vector flow $(\mathrm{GVF})$, respectively, as an external force. Figure [6] adds more results obtained by our approach for objects of complicated shape.

These and other experimental results show that the proposed stochastic deformable model outperforms other known deformable models in terms of both the overall accuracy and processing time. 


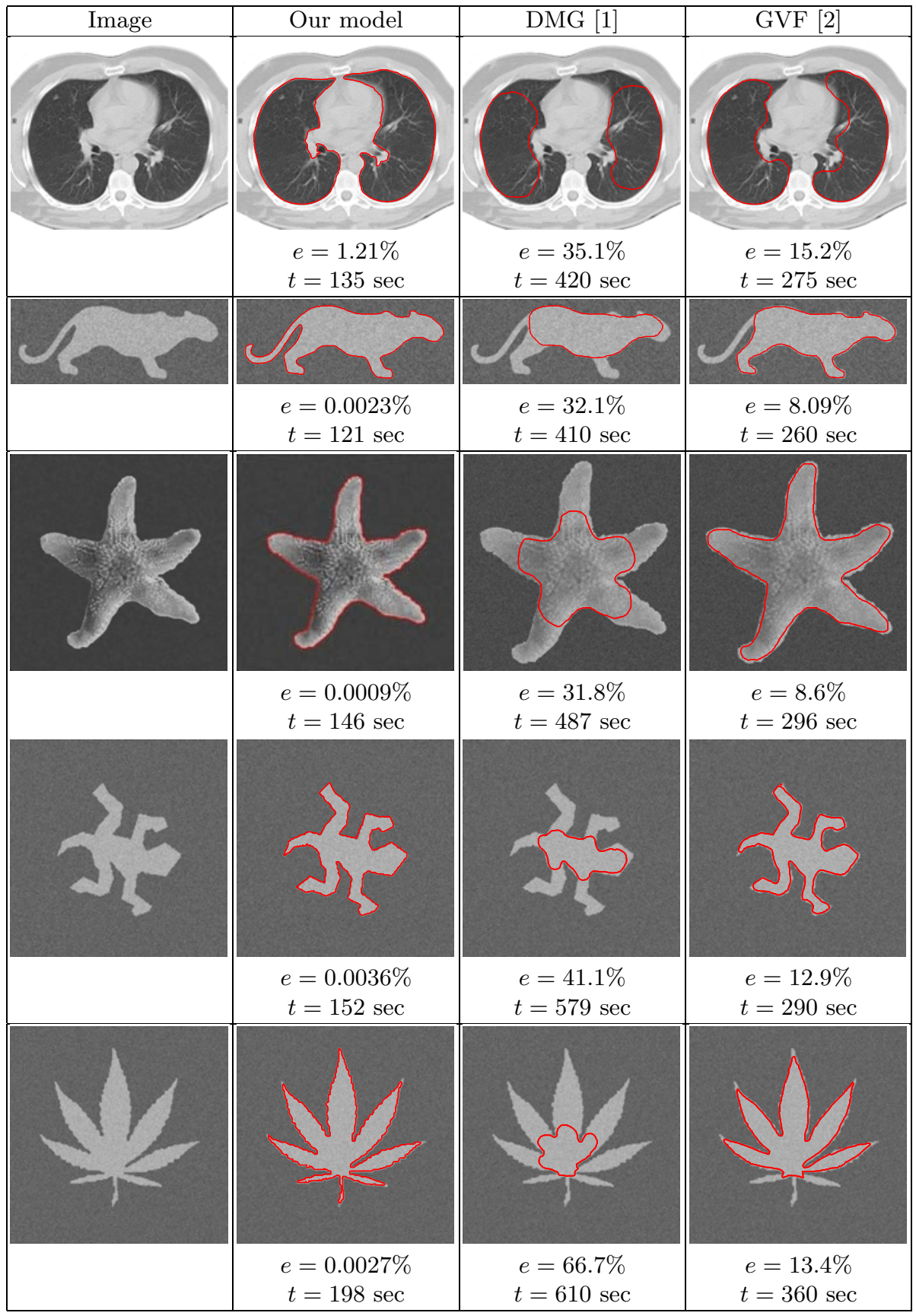

Fig. 5. Comparative results on various shapes $(e-$ the error; $t$ - time for segmentation; final object contours are shown in red) 


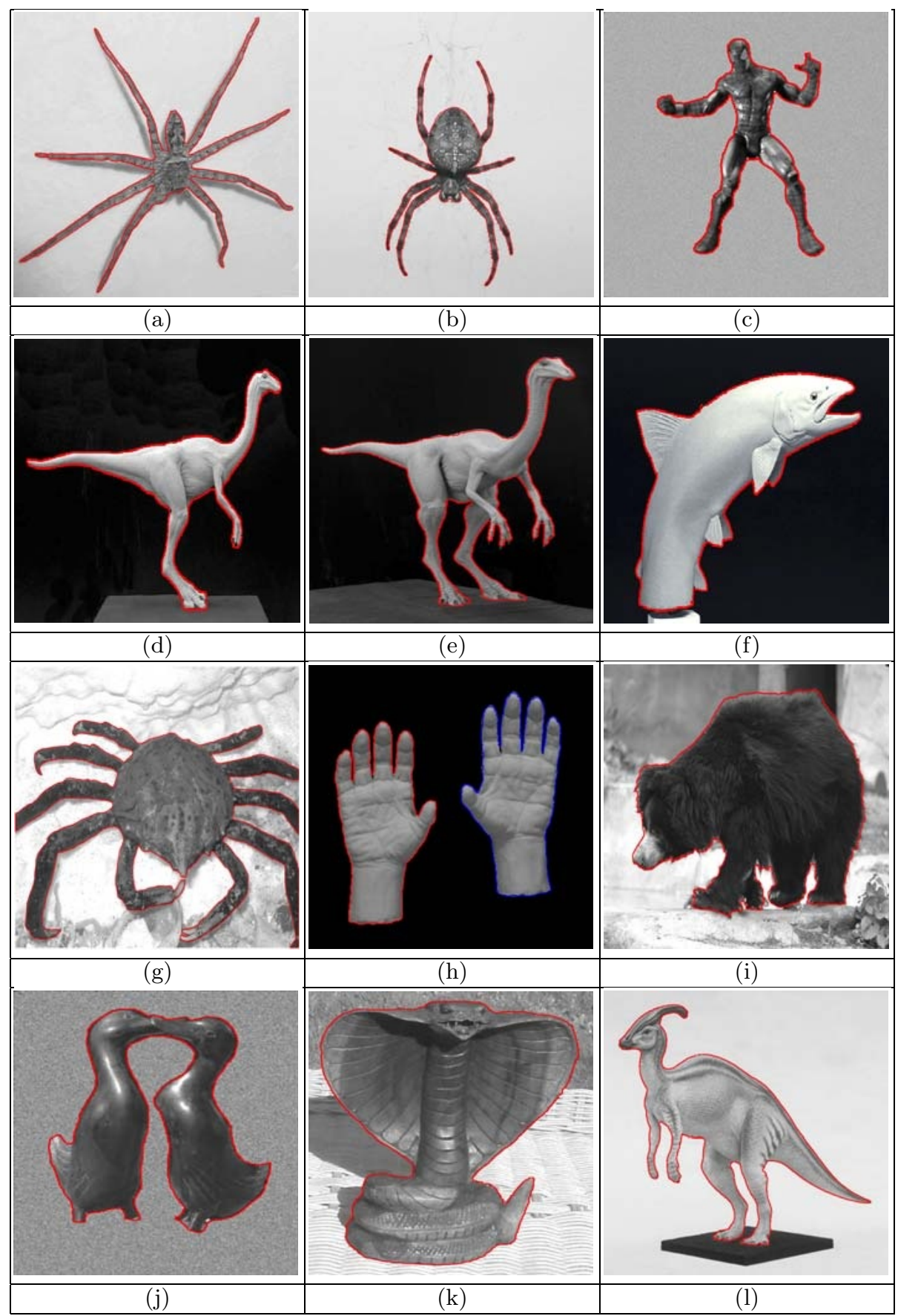

Fig. 6. More results obtained by our approach; final object contours are shown in red 


\section{References}

1. M. Kass, A. Witkin, and D. Terzopoulos, "Snakes: Active contour models," Int. J. Computer Vision, Vol. 1, pp. 321-331, 1987.

2. C. Xu and J.L. Prince, "Snakes, Shapes, and Gradient Vector Flow," IEEE Trans. Image Processing, Vol. 7, pp. 359-369, 1998.

3. N. Paragios and R. Deriche, "Geodesic Active Contours and Level Sets for the Detection and Tracking of Moving Objects," IEEE Trans. Pattern Analysis Machine Intelligence, Vol. 22, pp. 1-15, 2000.

4. N. Paragios, O. Mellina-Gottardo, and V. Armes, "Gradient vector flow fast geometric active contours," IEEE Trans. Pattern Analysis Machine Intelligence, Vol. 26, pp. 402-407, 2004.

5. A. Amini, T. Weymouth, and R. Jain, "Using dynamic programming for solving variational problems in vision," IEEE Trans. Pattern Analysis Machine Intelligence, vol. 12, pp. 855-867, 1990.

6. D.J. Williams and M. Shah, "A fast algorithm for active contours and curvature estimation," Proc. Int. Conf. Computer Vision, Graphics and Image Processing, Vol. 55, pp. 14-26, 1992.

7. R.P. Grzeszczuk and D.N. Levin, "Brownian strings: Segmenting images with stochastically deformable contours," IEEE Trans. Pattern Analysis Machine Intelligence, Vol. 19, pp. 1100-1114, 1997.

8. Yu. Boykov and V. Kolmogorov, "An experimental comparison of min-cut / maxflow algorithms," in: Proc. Third Int. Workshop Energy Minimization Methods in Computer Vision and Pattern Recogn., Sophia Antipolis, France, Sept. 2001 (Lecture Notes in Comp. Science 2134, Springer: Berlin, pp. 359-374, 2001.

9. Yu. Boykov and V. Kolmogorov, "Computing geodesics and minimal surfaces via graph cuts," in: Proc. IEEE Int. Conf. Computer Vision, Nice, France, Oct. 14-17, 2003, IEEE CS Press, pp. 26-33, 2003.

10. G. Gimel'farb, A. A. Farag, and A. El-Baz, "Expectation-Maximization for a linear combination of Gaussians," in Proc. IAPR Int. Conf. Pattern Recognition, Cambridge, UK, 23-26 Aug. 2004, IEEE CS Press, Vol. 3, 2004, pp. 422-425.

11. G. L. Gimel'farb, Image Textures and Gibbs Random Fields, Kluwer Academic, 1999.

12. A. A. Farag, A. El-Baz, and G. Gimelfarb, "Experimental Evaluation of Statitical and Deformable Model-Based Segmentation," TR-CVIP04-11, Computer Vision and Image Processing Laboratory, University of Louisville, KY, November 2004.

13. R. Goldenberg, R. Kimmel, E. Rivlin, and M. Rudzsky, "Fast Geodesic Active Contours," IEEE Trans. Image Processing, Vol. 10, no. 10, pp. 1467-1475, 2001. 